\title{
Algorithmic Annotation of Functional Roles for Components of 3,044 Human Molecular Pathways
}

\author{
Maxim Sorokin 1,2,3, Nicolas Borisov 1,3, Denis Kuzmin ${ }^{3}$, Alexander Gudkov², \\ Marianna Zolotovskaia ${ }^{3}$, Andrew Garazha ${ }^{1}$ and Anton Buzdin ${ }^{1,3,4,5 *}$
}

'Omicsway Corp., Walnut, CA, United States, ${ }^{2}$ Laboratory of Clinical Genomic Bioinformatics, I.M. Sechenov First Moscow State Medical University, Moscow, Russia, ${ }^{3}$ Laboratory for Translational Bioinformatics, Moscow Institute of Physics and Technology, Moscow, Russia, ${ }^{4}$ Laboratory of Systems Biology, Shemyakin-Ovchinnikov Institute of Bioorganic Chemistry, Moscow, Russia, ${ }^{5}$ World-Class Research Center "Digital Biodesign and Personalized Healthcare", Sechenov First Moscow State Medical University, Moscow, Russia

OPEN ACCESS

Edited by:

Yuriy L. Orlov,

I.M. Sechenov First Moscow State Medical University, Russia

Reviewed by:

Peter D'Eustachio,

New York University, United States

Pavel Kopnin,

Russian Cancer Research Center NN Blokhin, Russia

Teresa Bernadette Steinbichler, Innsbruck Medical University,

Austria

${ }^{*}$ Correspondence: Anton Buzdin

buzdin@oncobox.com bu3din@mail.ru

Specialty section:

This article was submitted to Computational Genomics, a section of the journal Frontiers in Genetics

Received: 13 October 2020 Accepted: 20 January 2021 Published: 09 February 2021

Citation:

Sorokin M, Borisov N, Kuzmin D,

Gudkov A, Zolotovskaia M,

Garazha A and Buzdin A (2021)

Algorithmic Annotation of Functional

Roles for Components of 3,044

Human Molecular Pathways.

Front. Genet. 12:617059.

doi: 10.3389/fgene.2021.617059
Current methods of high-throughput molecular and genomic analyses enabled to reconstruct thousands of human molecular pathways. Knowledge of molecular pathways structure and architecture taken along with the gene expression data can help interrogating the pathway activation levels (PALs) using different bioinformatic algorithms. In turn, the pathway activation profiles can characterize molecular processes, which are differentially regulated and give numeric characteristics of the extent of their activation or inhibition. However, different pathway nodes may have different functions toward overall pathway regulation, and calculation of PAL requires knowledge of molecular function of every node in the pathway in terms of its activator or inhibitory role. Thus, high-throughput annotation of functional roles of pathway nodes is required for the comprehensive analysis of the pathway activation profiles. We proposed an algorithm that identifies functional roles of the pathway components and applied it to annotate 3,044 human molecular pathways extracted from the Biocarta, Reactome, KEGG, Qiagen Pathway Central, NCl, and HumanCYC databases and including 9,022 gene products. The resulting knowledgebase can be applied for the direct calculation of the PALs and establishing large scale profiles of the signaling, metabolic, and DNA repair pathway regulation using high throughput gene expression data. We also provide a bioinformatic tool for PAL data calculations using the current pathway knowledgebase.

Keywords: functional algorithmic annotation, signaling pathways, DNA repair pathways, metabolic pathways, transcriptomics, proteomics, human molecular pathway regulation

\section{INTRODUCTION}

Intracellular molecular pathways are specific networks of interacting molecules that are involved in certain molecular functions (Junaid et al., 2020; Ma and Liao, 2020; Zheng et al., 2020). Knowledge of molecular pathways regulation is important for understanding intracellular processes related to all major events, including cell survival, growth, differentiation, motility, proliferation, senescence, malignization, and death (Buzdin et al., 2018). Molecular pathways are affected during organism growth and development, aging and disease progression (Parkhitko et al., 2020). Current methods of large-scale molecular and genomic analyses enabled to catalogue thousands of human molecular pathways (Wishart et al., 2020). In turn, highthroughput gene expression analyses like RNA sequencing (Sorokin et al., 2020a), expression 
microarrays (Schulze and Downward, 2001; Shih et al., 2005; Willier et al., 2013), or modern proteomic techniques (Buzdin et al., 2019) can provide adequate amounts of data to enable interactome-wide assessment of pathway activation.

Several popular algorithms and software like gene ontology (GO) analysis tools (Huang et al., 2009a,b), Metacore (Ekins et al., 2007) and Pathway Studio (Thomas and Bonchev, 2010) can analyze gene expression data to identify pathways significantly enriched by differentially regulated genes (Dubovenko et al., 2017). However, those techniques cannot identify the enhanced or inhibited status of a pathway regulation, because pathways may have numerous negative feedback loops or negative regulatory nodes (Khatri et al., 2012) and, therefore, the pathway nodes may involve both genes with its activating and genes with inhibitory functions (Borisov et al., 2020). Thus, upregulation of an inhibitory gene means pathway downregulation, and vice versa (Buzdin et al., 2018).

On the other hand, knowledge of the individual gene product roles within a pathway can make it readable in terms of finding its activation profiles. Indeed, several techniques had been proposed, e.g., Oncofinder (Buzdin et al., 2014b), iPANDA (Ozerov et al., 2016), and Oncobox (Borisov et al., 2020) that utilize transcriptome-wide or even proteome-wide (Borisov et al., 2017) data to calculate pathway activation levels (PALs). Those are the numeric characteristics that can be used in all types of comparisons including biomarker investigations. Overall, PALs were found to be superior cancer biomarkers compared to individual gene expression levels (Borisov et al., 2014; Lezhnina et al., 2014). A number of PALs were found to be characteristic for cancer drug response (Zhu et al., 2015) and sensitivity to X-ray irradiation (Sorokin et al., 2018), asthma (Alexandrova et al., 2016), Hutchinson-Gilford progeria (Aliper et al., 2015), macular degeneration (Makarev et al., 2014), fibrosis (Makarev et al., 2016), viral infection (Buzdin et al., 2016), and aging (Aliper et al., 2016). Algorithms were developed to convert pathway activation data into the optimized selection of cancer drugs (Artemov et al., 2015; Tkachev et al., 2020) that had several recent clinical applications (Poddubskaya et al., 2018, 2019a,b; Sorokin et al., 2020b). However, those studies used manually curated/annotated pathways and were, therefore, limited by the overall number ( 10 or $\sim 100)$ of pathways under analysis. Thus, it is important to annotate more pathways in a universal way to obtain a large-scale overview of the human interactome.
We proposed an algorithm that identifies functional roles of the pathway components based on the pathway topology and applied it here to annotate 3,044 human molecular pathways extracted from the Biocarta, Reactome, KEGG, NCI, and HumanCYC databases, collectively covering 9,022 gene products. The resulting knowledgebase can be applied for the direct calculation of the PALs and establishing large scale profiles of the signaling, metabolic, and DNA repair pathway regulation using high throughput gene expression data.

\section{RESULTS AND METHODS}

\section{Extraction of Molecular Pathway Data}

We extracted structures of molecular pathways from the National Cancer Institute (NCI; Schaefer et al., 2009), Biocarta (Nishimura, 2001), Qiagen Pathway Central, ${ }^{1}$ HumanCyC (Romero et al., 2004), Reactome (Croft et al., 2014), and Kyoto Encyclopedia of Genes and Genomes (KEGG; Kanehisa et al., 2010) databases (Table 1). For all the databases but Qiagen Pathway Central, the data on the pathway architecture, nodes and pairwise activation/inhibition interactions were extracted in biopax format. In the case of Qiagen Pathway Central database, no machine-readable format of data was available, and we manually curated data from the available graphical pathway representations (Table 1).

In addition to the extracted full-size pathways, we also generated a number of subsequent "micropathways" that were derivatives of the complete pathways (Figures 1A,B) Micropathway is a sub-graph, which contains "molecular function" node and nodes from all possible paths of length 3 including terminal "molecular function" node. Many full-size pathways have two or more terminal branches that may have different functional impact(s). We, therefore, introduced micropathways to characterize molecular processes in more detail by separately analyzing different terminal branches of the pathways. Totally, we processed 3,044 pathways including 2018 full-size, or "core" pathways, and 1,026 micropathways that covered collectively products of 9,022 human genes (Table $\mathbf{1}$ ).

${ }^{1}$ https://www.qiagen.com/gb/resources/resourcedetail?id=5869e38a-5033-4ccb-a281d869893acf4e\&lang=en

TABLE 1 | Statistics of the curated pathway databases.

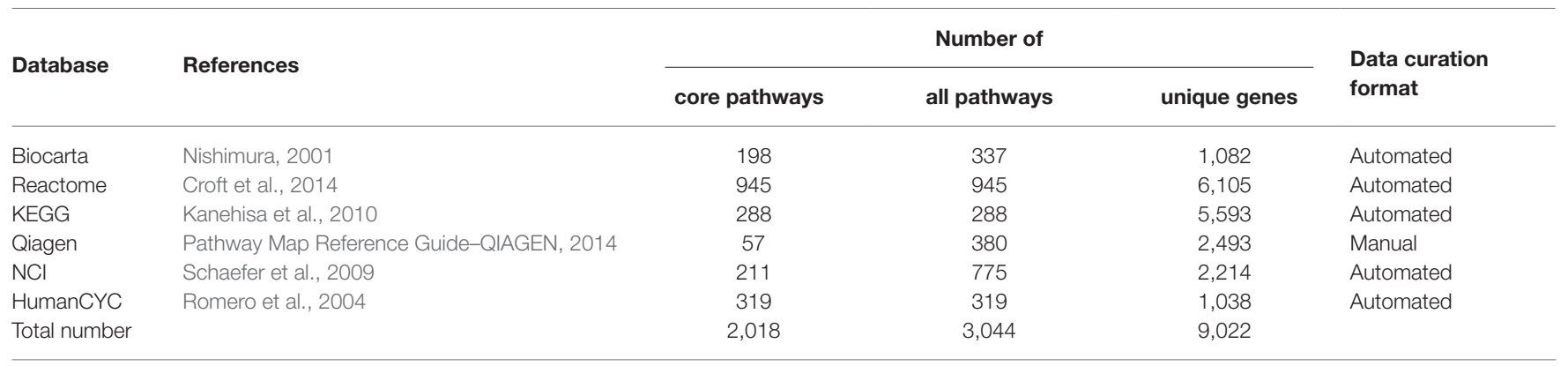

Number of all pathways includes core pathways and micropathways. Number of unique gene products covered by pathways from the respective database. For total number, the amount of unique gene products for all pathways is shown. 
Note that number of pathway nodes was smaller than the number of genes involved in a pathway because one node could correspond to several gene products.

For several pathway components alternative gene names were used and we then converted all gene names according to the Human Genome Organization HGNC nomenclature (Povey et al., 2001).

\section{Algorithmic Annotation of Molecular Pathways}

For most of the published PAL applications, maximum five types of functional roles for gene products were comprised. These roles, described by an Activator/Repressor Role (ARR) parameter can be formulated as follows: pathway activator $(A R R=1)$, rather activator $(A R R=0.5)$, repressor $(A R R=-1)$, rather repressor $(\mathrm{ARR}=-0.5)$, and gene product with uncertain or inconsistent role $(A R R=0)$. In the previous studies, ARR values were obtained by manually curating pathway graphs. This is however not feasible for annotating thousands of molecular pathways. We developed an original algorithm that automatically assigns ARR score values to gene products that participate in a molecular pathway.

The ARR annotation algorithm is based on the machine reading of gene product interaction graph within each pathway. Nodes correspond to gene products, and the ribs between every pair of nodes represent molecular interaction between the corresponding gene products. Each rib on the graph has a direction and is characterized by an activator or inhibitory nature of the molecular interaction it represents. For the correct calculation of ARR values, the pathway graph must be connected, wherein a weak connectivity is acceptable.

If the pathway molecular interaction graph meets these criteria, then ARR coefficients can be algorithmically assigned to the participating gene products. For the biochemical pathways, we put enzyme gene names on the pathway nodes, and the interaction ribs represented directions of the catalyzed reactions.

The algorithm used consisted of the following major steps.

i. Initialization. At this stage, a major node is algorithmically identified to be the "central" node of the pathway graph (Figure 1C). The major node will be used as the standard of pathway function with ARR $=1$. To identify the central node, for every pathway node $(\mathrm{V})$ two parameters $\mathrm{N}$ and $\mathrm{M}$ are calculated where $\mathrm{N}$ is the number of other nodes, which can be reached when moving from the node V, and $\mathrm{M}$ is the number of other nodes from which the node $\mathrm{V}$ can be reached. N+M, therefore, is the number of other nodes that are directly connected with the node $\mathrm{V}$. The central node will be the node $V_{\max }$ for which $N+M$ reaches the maximum value. The central node identified is then assigned with $\mathrm{ARR}=1$ value. It serves as the starting point for further recursive assignment of ARR values to the other nodes. If multiple nodes have the same maximal $\mathrm{N}+\mathrm{M}$, then V-node for a pathway is selected randomly among those "maximal" nodes. Therefore, the algorithm is suitable also for circular-organized pathways, where all nodes will have equal $\mathrm{N}+\mathrm{M}$. ii. Recursion. For every node $\mathrm{V}$, all connected nodes $\mathrm{P}_{\mathrm{i}}$ under ARR annotation may have ribs either directed toward $\mathrm{V}$ $(\mathrm{Pi} \rightarrow \mathrm{V})$ or outward $\mathrm{V}(\mathrm{Pi} \leftarrow \mathrm{V})$ on the graph. During recursion, each rib can be considered only once in order to prevent endless recursion in case of cyclic interactions on the graph. If the rib has an "activator" characteristic, temporary ARRtemp $=1$ is assigned to the node $P_{\mathrm{i}}$. In contrast, if the rib has an "inhibitor" characteristic, $P_{i}$ is assigned with $\mathrm{ARR}_{\text {temp }}=-1$. Conversely, all the gene products included in the node $P_{i}$ receive the same $A R R_{\text {temp }}$ characteristics.

Let gene product $\mathrm{GP}_{\mathrm{i}}$ belongs to node $\mathrm{P}_{\mathrm{i}}$. If $\mathrm{GP}_{\mathrm{i}}$ was never previously considered in the graph traversal, $A R R=\mathrm{ARR}_{\text {temp }(\mathrm{P} \mathrm{Pi})}$ for the node $\mathrm{P}_{\mathrm{i}}$ would be assigned for $\mathrm{GP}_{\mathrm{i}}$. In the case when $\mathrm{GP}_{\mathrm{i}}$ was previously considered in the graph traversal and the previously assigned ARR of it node is equal to the current $A R R_{\text {temp }(\mathrm{Pi})}$ then $\mathrm{ARR}=A \mathrm{AR}_{\text {temp }}$ would be assigned to the node $\mathrm{P}_{\mathrm{i}}$. If $\mathrm{GP}_{\mathrm{i}}$ was previously considered in the graph traversal but its previously assigned $A R R$ is not equal to $A R R_{\text {temp }(\mathrm{P} i)}$, then $A R R$ is assigned to the gene product $\mathrm{GP}_{\mathrm{i}}$ according to the following conflict resolution rule.

If a gene product $\mathrm{GP}_{\mathrm{i}}$ with previously specified ARR or ARRs is currently considered in the graph traversal but its previously assigned $\operatorname{ARR}(\mathrm{s})$ contradict(s) with the $\mathrm{ARR}_{\text {temp(Pi) }}$, then the conflict(s) should be resolved as follows:

1. If the signs of the previous $A R R$ coefficient $(s)$ and $A R R_{\text {temp }(\mathrm{P} i)}$ are different, then the resulting $A R R_{\text {final( }(\mathrm{Pi})}=0$;

2. If the difference between $A R R_{\text {temp }(\mathrm{Pi})}$ and any of the previous $\mathrm{ARRs}_{(\mathrm{GP})}$ does not exceed 0.5 and at least one of the ARRs is positive, the resulting $\mathrm{ARR}_{\text {final(Pi) }}=0.5$;

3. If the difference between $\mathrm{ARR}_{\text {temp }(\mathrm{Pi})}$ and any of the previous $\mathrm{ARRs}_{(\mathrm{GP})}$ does not exceed 0.5 and at least one of the ARRs is negative, the resulting $\mathrm{ARR}_{\text {final }\left(\mathrm{P}_{\mathrm{i}}\right)}=-0.5$.

Then the recursion $R$ is initiated for every node $P_{i}$ all of its gene products starting from the nodes proximate to the central node $\mathrm{V}$. As a result, the algorithm will assign ARR values to all the connected the graph nodes and the enclosed gene products.

After the recursion finalization pathway activators will have $\mathrm{ARR}=1$, rather activators - ARR $=0.5$, inhibitors $\mathrm{ARR}=-1$, rather inhibitors $-\mathrm{ARR}=-0.5$, and genes with inconsistent role $-\mathrm{ARR}=0$. The recursion is stopped when a vertex with $0,0.5$, or -0.5 ARR is encountered during the traversal of the graph. This rule is needed because otherwise all vertices will have ARR $0,0.5$, or -0.5 in case of the only one ARR inconsistency found. However, this rule also may lead to exclusion of some genes described in the original source.

Therefore, the gene products included in the molecular pathway database will have the assigned ARR values representing their functional significances in the given molecular pathway. These values can be used for further calculations of the PALs according to any algorithm of PAL calculation.

\section{Annotated Pathways Knowledgebase}

We report here an ARR-curated database of 3,044 molecular pathways including 2,018 core pathways and 1,026 micropathways 


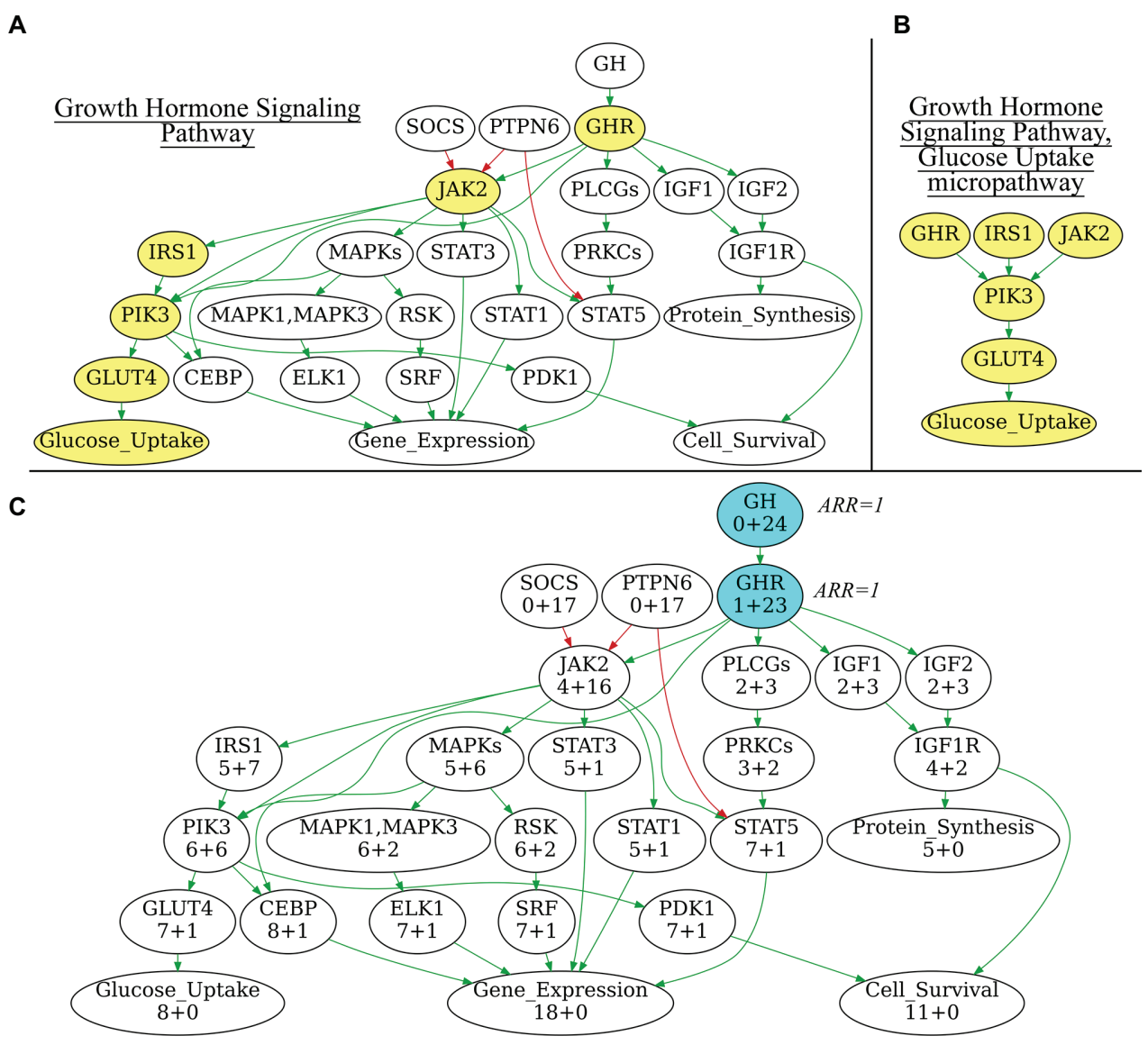

FIGURE 1 | (A) Growth Hormone Signaling Pathway with highlighted Glucose Uptake micropathway. (B) Glucose Uptake micropathway obtained from Growth Hormone Signaling Pathway. (C) N+M values for all vertices of Growth Hormone Signaling Pathway graph. The vertices with maximal N+M values are highlighted in blue, these vertices are equal major node candidates and get Activator/Repressor Role (ARR) = 1. Different edge colors indicate edge attribute: green is for "activation," red is for "inhibition." Structure of the Growth Hormone Signaling Pathway is derived from Qiagen Pathway Central. Yellow vertices on panel 1A indicate micropathway Glucose Uptake within Growth Hormone Signaling Pathway.

(Supplementary Dataset 1). The current pathway name reflects its source database and its name in the source database. For every pathway, there is a separate .csv file including the following three worksheets: (i) genes, (ii) edges, and (iii) nodes. The worksheet (i) genes include gene names according to HUGO Gene Nomenclature Committee (HGNC) nomenclature and the corresponding ARRs for the gene products participating in the pathway under consideration. The worksheet (ii) edges include information about molecular interactions between every pair of the interacting pathway nodes. Every node is defined by the names of gene products or physiological outcome(s) that form this node. The interaction type is specified as "activation," "inhibition," or "undefined," where appropriate. The worksheet (iii) nodes include node names and gene names corresponding to every node on the pathway graph.

It should be noted that annotation of similar pathways may be different between the source databases. For example, EGFR signaling pathway is presented in Qiagen database as "EGF_ Pathway," in Reactome as "reactome_Signaling_by_EGFR_Main_ Pathway" and in Biocarta as "biocarta_egf_signaling_Main_Pathway."
Yet conceptually similar, all three pathways have different gene and edge compositions. In this study, we did not aim to identify inconsistences between different source databases and annotated all the pathways under their original names.

We made freely accessible software for PAL calculation using the annotated pathway database accessible following the link: https://pypi.org/project/oncoboxlib/. Algorithm is implemented as a Python library. It takes normalized (by DESeq2, quantile normalization or other) gene expression data as an input. Gene symbols should be provided in HGNC format accessible through the web-site genenames.org. At least two groups of samples are required: cases and controls, each group represented by at least one sample. Sample names should contain "Norm_" (for controls) or "Tumour_" (for cases). Output will contain PAL values for each pathway in each sample. All annotated pathway datasets mentioned in this paper alternatively can be downloaded and used for PAL calculation using the same link. ${ }^{2}$

${ }^{2 h t t p s: / / p y p i . o r g / p r o j e c t / o n c o b o x l i b / ~}$ 
We also provide here an example of PAL calculation for real-world data. We extracted gene expression data for gastric cancer samples ( $n=16$; Sorokin et al., 2020b) together with gene expression profiles of healthy stomach $(n=7)$ samples of patients who died in road accidents (Suntsova et al., 2019), that were sequenced using the same equipment and protocols. Cancerous samples were marked as "Tumour_" and normal samples - as "Norm_." Then we calculated PAL values (3,044 for each sample) for all molecular profiles using the above software, which produced an output file "pal.csv" (Supplementary Dataset 1).

\section{DISCUSSION}

We propose here the recursive algorithm for functional annotation of the molecular pathway nodes, and its application to annotation of 3,044 human molecular pathways, including signaling, metabolic, and DNA repair pathways extracted from six major pathway hubs (Table 1). The ARR-annotated pathways can be used for further calculations of PALs using highthroughput gene expression data, e.g., RNA sequencing or proteomic profiles (Buzdin et al., 2018; Figure 2). To this end, several previously published bioinformatic methods can be employed (Buzdin et al., 2014a; Ozerov et al., 2016; Borisov et al., 2020), and the PAL values returned can be applied for a variety of applications including fundamental research (Pasteuning-Vuhman et al., 2017), drug development (Aliper et al., 2017a; Ravi et al., 2018; Bakula et al., 2019), and personalized medicine (Poddubskaya et al., 2019a; Moisseev et al., 2020). Technically, PAL values can be used as the next-generation molecular biomarkers (Aliper et al., 2017b; Borisov et al., 2017; Sorokin et al., 2020c) or as the substrates for various machine learning applications (Borisov et al., 2018; Tkachev et al., 2018).

The proposed algorithm is suitable for the analysis of pathways with already established gene content and known topology of its molecular components. The algorithm can be used for agnostic

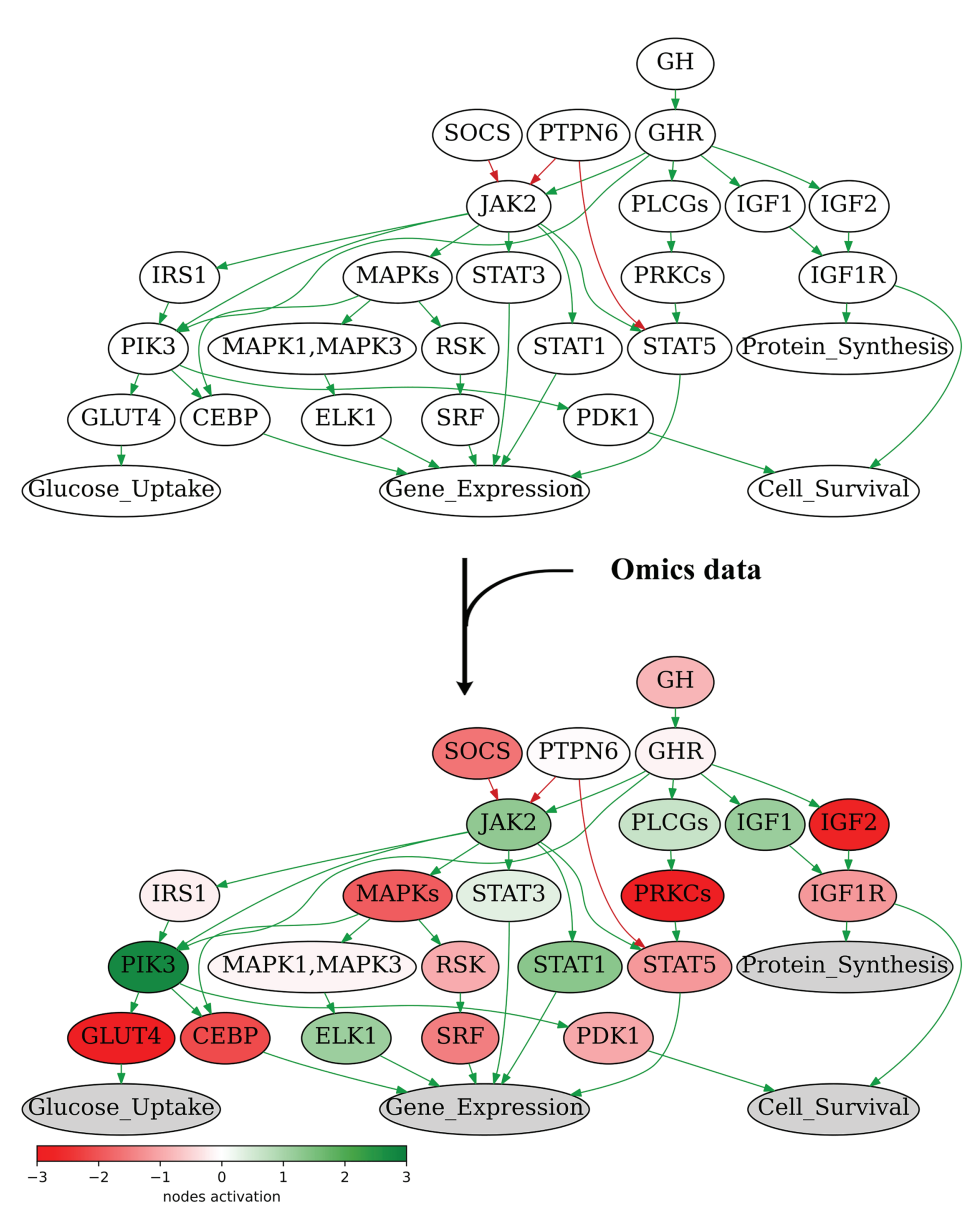

FIGURE 2 | Node activation of Growth Hormone Signaling Pathway for gastric cancer sample GC.11_S19_R1_001 from Sorokin et al. (2020b). Node activation is a sum of logarithmic case-to-norm ratio (CNR) for all genes in the node. CNR is ratio of expression levels in tumor sample and averaged normal sample. The RNA sequencing tumor profile (gastric cancer) was obtained from Sorokin et al. (2020b). The RNA sequencing profiles of normal gastric tissue were obtained from Oncobox Atlas of Normal Tissue Expression (ANTE) data (Suntsova et al., 2019). Different edge colors indicate edge attribute: green is for "activation," red is for "inhibition." Structure of the Growth Hormone Signaling Pathway is derived from Qiagen Pathway Central. 
objective characterization of interacting gene networks. The underlying rationale allows reducing operator's errors and subjectivity in annotating the molecular roles of pathway components, which are inevitable in case of manual curation of the pathway graphs including hundreds of nodes. Another advantage is the pathwaycentric approach during annotation, when gene product role in one pathway can be different from its role in another pathway.

The major limitations deal with the algorithm applicability only for the tasks of further calculations of pathway activation scores/ranks. Such an approach also does not address the issue of crosstalk between different molecular pathways, because all pathways are analyzed separately.

In this study, we annotated a collection of previously published human molecular pathways (Supplementary Dataset 1). We plan to update the current human knowledgebase annually with new releases of already included datasets and addition of new pathway collections, e.g., recently published by Wishart et al. (2020). However, the method proposed here can be used to characterize any new set of molecular pathways with the connectivity and pairwise nodes activation/inhibition information not only for the human interactome, but also for the other biological objects under investigation.

\section{DATA AVAILABILITY STATEMENT}

The original contributions presented in the study are included in the article/Supplementary Material, further inquiries can be directed to the corresponding author.

\section{REFERENCES}

Alexandrova, E., Nassa, G., Corleone, G., Buzdin, A., Aliper, A. M., Terekhanova, N., et al. (2016). Large-scale profiling of signalling pathways reveals an asthma specific signature in bronchial smooth muscle cells. Oncotarget 7, 25150-25161. doi: 10.18632/oncotarget.7209

Aliper, A., Belikov, A. V., Garazha, A., Jellen, L., Artemov, A., Suntsova, M., et al. (2016). In search for geroprotectors: in silico screening and in vitro validation of signalome-level mimetics of young healthy state. Aging 8, 2127-2152. doi: 10.18632/aging.101047

Aliper, A. M., Csoka, A. B., Buzdin, A., Jetka, T., Roumiantsev, S., Moskalev, A., et al. (2015). Signaling pathway activation drift during aging: HutchinsonGilford Progeria Syndrome fibroblasts are comparable to normal middle-age and old-age cells. Aging 7, 26-37. doi: 10.18632/aging.100717

Aliper, A., Jellen, L., Cortese, F., Artemov, A., Karpinsky-Semper, D., Moskalev, A., et al. (2017a). Towards natural mimetics of metformin and rapamycin. Aging 9, 2245-2268. doi: 10.18632/aging.101319

Aliper, A. M., Korzinkin, M. B., Kuzmina, N. B., Zenin, A. A., Venkova, L. S., Smirnov, P. Y., et al. (2017b). Mathematical justification of expression-based pathway activation scoring (PAS). Methods Mol. Biol. 1613, 31-51. doi: 10.1007/978-1-4939-7027-8_3

Artemov, A., Aliper, A., Korzinkin, M., Lezhnina, K., Jellen, L., Zhukov, N., et al. (2015). A method for predicting target drug efficiency in cancer based on the analysis of signaling pathway activation. Oncotarget 6, 29347-29356. doi: 10.18632/oncotarget.5119

Bakula, D., Ablasser, A., Aguzzi, A., Antebi, A., Barzilai, N., Bittner, M. I., et al. (2019). Latest advances in aging research and drug discovery. Aging 11, 9971-9981. doi: 10.18632/aging.102487

Borisov, N., Sorokin, M., Garazha, A., and Buzdin, A. (2020). Quantitation of molecular pathway activation using RNA sequencing data. Methods Mol. Biol. 2063, 189-206. doi: 10.1007/978-1-0716-0138-9_15

\section{AUTHOR CONTRIBUTIONS}

MS, NB, DK, and $\mathrm{AB}$ contributed to conception and design of the study. MS developed recursive pathway annotation algorithm. DK, AGu, MZ, and AGa manually curated the pathways and performed recursive algorithm implementations. $\mathrm{AB}, \mathrm{MZ}$, and MS wrote the manuscript. All authors contributed to the article and approved the submitted version.

\section{FUNDING}

This study was supported by the Amazon and Microsoft Azure grants for cloud-based computational facilities. Financial support was provided by the Russian Foundation for basic research grant 19-29-01108.

\section{ACKNOWLEDGMENTS}

We thank Oncobox/OmicsWay research program in machine learning and digital oncology for software and starting pathway databases for this study.

\section{SUPPLEMENTARY MATERIAL}

The Supplementary Material for this article can be found online at: https://www.frontiersin.org/articles/10.3389/fgene.2021.617059/ full\#supplementary-material

Borisov, N., Suntsova, M., Sorokin, M., Garazha, A., Kovalchuk, O., and Aliper, A. et al. (2017). Data aggregation at the level of molecular pathways improves stability of experimental transcriptomic and proteomic data. Cell Cycle 16, 1810-1823. doi: 10.1080/15384101.2017.1361068

Borisov, N., Tkachev, V., Suntsova, M., Kovalchuk, O., Zhavoronkov, A., Muchnik, I., et al. (2018). A method of gene expression data transfer from cell lines to cancer patients for machine-learning prediction of drug efficiency. Cell Cycle 17, 486-491. doi: 10.1080/15384101.2017.1417706

Borisov, N. M., Terekhanova, N. V., Aliper, A. M., Venkova, L. S., Smirnov, P. Y., Roumiantsev, S., et al. (2014). Signaling pathways activation profiles make better markers of cancer than expression of individual genes. Oncotarget 5, 10198-10205. doi: 10.18632/oncotarget.2548

Buzdin, A. A., Artcibasova, A. V., Fedorova, N. F., Suntsova, M. V., Garazha, A. V., Sorokin, M. I., et al. (2016). Early stage of cytomegalovirus infection suppresses host microRNA expression regulation in human fibroblasts. Cell Cycle 15, 3378-3389. doi: 10.1080/15384101.2016.1241928

Buzdin, A., Sorokin, M., Garazha, A., Glusker, A., Aleshin, A., Poddubskaya, E., et al. (2019). RNA sequencing for research and diagnostics in clinical oncology. Semin. Cancer Biol. 60, 311-323. doi: 10.1016/j.semcancer.2019.07.010

Buzdin, A., Sorokin, M., Garazha, A., Sekacheva, M., Kim, E., Zhukov, N., et al. (2018). Molecular pathway activation - new type of biomarkers for tumor morphology and personalized selection of target drugs. Semin. Cancer Biol. 53, 110-124. doi: 10.1016/j.semcancer.2018.06.003

Buzdin, A. A., Zhavoronkov, A. A., Korzinkin, M. B., Roumiantsev, S. A., Aliper, A. M., Venkova, L. S., et al. (2014a). The OncoFinder algorithm for minimizing the errors introduced by the high-throughput methods of transcriptome analysis. Front. Mol. Biosci. 1:8. doi: 10.3389/fmolb.2014.00008

Buzdin, A. A., Zhavoronkov, A. A., Korzinkin, M. B., Venkova, L. S., Zenin, A. A., Smirnov, P. Y., et al. (2014b). Oncofinder, a new method for the analysis of intracellular signaling pathway activation using transcriptomic data. Front. Genet. 5:55. doi: 10.3389/fgene.2014.00055 
Croft, D., Mundo, A. F., Haw, R., Milacic, M., Weiser, J., Wu, G., et al. (2014). The Reactome pathway knowledgebase. Nucleic Acids Res. 42, D472-D477. doi: $10.1093 /$ nar/gkt1102

Dubovenko, A., Nikolsky, Y., Rakhmatulin, E., and Nikolskaya, T. (2017). "Functional analysis of OMICs data and small molecule compounds in an integrated "knowledge-based" platform" in Methods in molecular biology. eds. T. V. Tatarinova and Y. Nikolsky (Humana Press Inc.), 101-124.

Ekins, S., Nikolsky, Y., Bugrim, A., Kirillov, E., and Nikolskaya, T. (2007). Pathway mapping tools for analysis of high content data. Methods Mol. Biol. 356, 319-350. doi: 10.1385/1-59745-217-3:319

Huang, D. W., Sherman, B. T., and Lempicki, R. A. (2009a). Bioinformatics enrichment tools: paths toward the comprehensive functional analysis of large gene lists. Nucleic Acids Res. 37, 1-13. doi: 10.1093/nar/gkn923

Huang, D. W., Sherman, B. T., and Lempicki, R. A. (2009b). Systematic and integrative analysis of large gene lists using DAVID bioinformatics resources. Nat. Protoc. 4, 44-57. doi: 10.1038/nprot.2008.211

Junaid, M., Akter, Y., Afrose, S. S., Tania, M., and Khan, M. A. (2020). Biological role of $\mathrm{AKT}$, and regulation of AKT signaling pathway by thymoquinone: perspectives in cancer therapeutics. Mini Rev. Med. Chem. 20. doi: 10.217 4/1389557520666201005143818 [Epub ahead of print]

Kanehisa, M., Goto, S., Furumichi, M., Tanabe, M., and Hirakawa, M. (2010). $\{$ KEGG\} for representation and analysis of molecular networks involving diseases and drugs. Nucleic Acids Res. 38, D355-D360. doi: 10.1093/nar/ gkp896

Khatri, P., Sirota, M., and Butte, A. J. (2012). Ten years of pathway analysis: current approaches and outstanding challenges. PLoS Comput. Biol. 8:e1002375. doi: 10.1371/journal.pcbi.1002375

Lezhnina, K., Kovalchuk, O., Zhavoronkov, A. A., Korzinkin, M. B., Zabolotneva, A. A., Shegay, P. V., et al. (2014). Novel robust biomarkers for human bladder cancer based on activation of intracellular signaling pathways. Oncotarget 5, 9022-9032. doi: 10.18632/oncotarget.2493

Ma, C. -Y., and Liao, C. -S. (2020). A review of protein-protein interaction network alignment: from pathway comparison to global alignment. Comput. Struct. Biotechnol. J. 18, 2647-2656. doi: 10.1016/j.csbj.2020.09.011

Makarev, E., Cantor, C., Zhavoronkov, A., Buzdin, A., Aliper, A., and Csoka, A. B. (2014). Pathway activation profiling reveals new insights into Age-related Macular Degeneration and provides avenues for therapeutic interventions. Aging 6, 1064-1075. doi: 10.18632/aging.100711

Makarev, E., Izumchenko, E., Aihara, F., Wysocki, P. T., Zhu, Q., Buzdin, A., et al. (2016). Common pathway signature in lung and liver fibrosis. Cell Cycle 15, 1667-1673. doi: 10.1080/15384101.2016.1152435

Moisseev, A., Albert, E., Lubarsky, D., Schroeder, D., and Clark, J. (2020). Transcriptomic and genomic testing to guide individualized treatment in chemoresistant gastric cancer case. Biomedicine 8:67. doi: 10.3390/biomedicines 8030067

Nishimura, D. (2001). BioCarta. Biotech Softw. Internet Rep. 2, 117-120. doi: $10.1089 / 152791601750294344$

Ozerov, I. V., Lezhnina, K. V., Izumchenko, E., Artemov, A. V., Medintsev, S., Vanhaelen, Q., et al. (2016). In silico Pathway Activation Network Decomposition Analysis (iPANDA) as a method for biomarker development. Nat. Commun. 7:13427. doi: 10.1038/ncomms13427

Parkhitko, A. A., Filine, E., Mohr, S. E., Moskalev, A., and Perrimon, N. (2020). Targeting metabolic pathways for extension of lifespan and healthspan across multiple species. Ageing Res. Rev. 64:101188. doi: 10.1016/j.arr.2020. 101188

Pasteuning-Vuhman, S., Boertje-Van Der Meulen, J. W., Van Putten, M., Overzier, M., Ten Dijke, P., Kiełbasa, S. M., et al. (2017). New function of the myostatin/activin type I receptor (ALK4) as a mediator of muscle atrophy and muscle regeneration. FASEB J. 31, 238-255. doi: 10.1096/ f. $201600675 \mathrm{R}$

Pathway Map Reference Guide-QIAGEN (2014). Available at: https://www. qiagen.com/gb/resources/resourcedetail? $\mathrm{id}=5869 \mathrm{e} 38 \mathrm{a}-5033-4 \mathrm{ccb}-\mathrm{a} 281$ d869893acf4e\&lang=en (Accessed October 12, 2020).

Poddubskaya, E. V., Baranova, M. P., Allina, D. O., Sekacheva, M. I., Makovskaia, L. A., Kamashev, D. E., et al. (2019b). Personalized prescription of imatinib in recurrent granulosa cell tumor of the ovary: case report. Mol. Case Stud. 5:mcs.a003434. doi: 10.1101/mcs.a003434

Poddubskaya, E. V., Baranova, M. P., Allina, D. O., Smirnov, P. Y., Albert, E. A., Kirilchev, A. P., et al. (2018). Personalized prescription of tyrosine kinase inhibitors in unresectable metastatic cholangiocarcinoma. Exp. Hematol. Oncol. 7:21. doi: 10.1186/s40164-018-0113-x

Poddubskaya, E., Bondarenko, A., Boroda, A., Zotova, E., Glusker, A., Sletina, S., et al. (2019a). Transcriptomics-guided personalized prescription of targeted therapeutics for metastatic ALK-positive lung cancer case following recurrence on ALK inhibitors. Front. Oncol. 9:1026. doi: 10.3389/fonc.2019.01026

Povey, S., Lovering, R., Bruford, E., Wright, M., Lush, M., and Wain, H. (2001). The HUGO gene nomenclature committee (HGNC). Hum. Genet. 109, 678-680. doi: 10.1007/s00439-001-0615-0

Ravi, R., Noonan, K. A., Pham, V., Bedi, R., Zhavoronkov, A., Ozerov, I. V., et al. (2018). Bifunctional immune checkpoint-targeted antibody-ligand traps that simultaneously disable TGF $\beta$ enhance the efficacy of cancer immunotherapy. Nat. Commun. 9:741. doi: 10.1038/s41467-017-02696-6

Romero, P., Wagg, J., Green, M. L., Kaiser, D., Krummenacker, M., and Karp, P. D. (2004). Computational prediction of human metabolic pathways from the complete human genome. Genome Biol. 6:R2. doi: 10.1186/gb2004-6-1-r2

Schaefer, C. F., Anthony, K., Krupa, S., Buchoff, J., Day, M., Hannay, T., et al. (2009). PID: the pathway interaction database. Nucleic Acids Res. 37, D674-D679. doi: $10.1093 /$ nar/gkn653

Schulze, A., and Downward, J. (2001). Navigating gene expression using microarrays - a technology review. Nat. Cell Biol. 3, E190-E195. doi: $10.1038 / 35087138$

Shih, W., Chetty, R., and Tsao, M. -S. (2005). Expression profiling by microarrays in colorectal cancer (review). Oncol. Rep. 13, 517-524. doi: 10.3892/or.13.3.517

Sorokin, M., Ignatev, K., Barbara, V., Vladimirova, U., Muraveva, A., Suntsova, M., et al. (2020c). Molecular pathway activation markers are associated with efficacy of trastuzumab therapy in metastatic HER2-positive breast cancer better than individual gene expression levels. Biochemistry 85, 758-772. doi: 10.1134/S0006297920070044

Sorokin, M., Ignatev, K., Poddubskaya, E., Vladimirova, U., Gaifullin, N., Lantsov, D., et al. (2020a). RNA sequencing in comparison to immunohistochemistry for measuring cancer biomarkers in breast cancer and lung cancer specimens. Biomedicine 8:114. doi: 10.3390/BIOMEDICIN ES8050114

Sorokin, M., Kholodenko, R., Grekhova, A., Suntsova, M., Pustovalova, M., Vorobyeva, N., et al. (2018). Acquired resistance to tyrosine kinase inhibitors may be linked with the decreased sensitivity to X-ray irradiation. Oncotarget 9, 5111-5124. doi: 10.18632/oncotarget.23700

Sorokin, M., Poddubskaya, E., Baranova, M., Glusker, A., Kogoniya, L., Markarova, E., et al. (2020b). RNA sequencing profiles and diagnostic signatures linked with response to ramucirumab in gastric cancer. Cold Spring Harb. Mol. Case Stud. 6:mcs.a004945. doi: 10.1101/mcs. a004945

Suntsova, M., Gaifullin, N., Allina, D., Reshetun, A., Li, X., Mendeleeva, L., et al. (2019). Atlas of RNA sequencing profiles for normal human tissues. Sci. Data 6:36. doi: 10.1038/s41597-019-0043-4

Thomas, S., and Bonchev, D. (2010). A survey of current software for network analysis in molecular biology. Hum. Genomics 4, 353-360. doi: 10.1186/14797364-4-5-353

Tkachev, V., Sorokin, M., Garazha, A., Borisov, N., and Buzdin, A. (2020). "Oncobox method for scoring efficiencies of anticancer drugs based on gene expression data" in Methods in molecular biology. eds. K. Astakhova and S. A. Bukhari (Humana Press Inc.), 235-255.

Tkachev, V., Sorokin, M., Mescheryakov, A., Simonov, A., Garazha, A., Buzdin, A., et al. (2018). FLOating-window projective separator (FloWPS): a data trimming tool for support vector machines (SVM) to improve robustness of the classifier. Front. Genet. 9:717. doi: 10.3389/fgene.2018.00717

Willier, S., Butt, E., and Grunewald, T. G. P. (2013). Lysophosphatidic acid (LPA) signalling in cell migration and cancer invasion: a focussed review and analysis of LPA receptor gene expression on the basis of more than 1700 cancer microarrays. Biol. Cell. 105, 317-333. doi: 10.1111/boc. 201300011

Wishart, D. S., Li, C., Marcu, A., Badran, H., Pon, A., Budinski, Z., et al. (2020). PathBank: a comprehensive pathway database for model organisms. Nucleic Acids Res. 48, D470-D478. doi: 10.1093/nar/gkz861

Zheng, J., Yu, H., Zhou, A., Wu, B., Liu, J., Jia, Y., et al. (2020). It takes two to tango: coupling of Hippo pathway and redox signaling in biological process. Cell Cycle 19, 1-16. doi: 10.1080/15384101.2020.1824448 
Zhu, Q., Izumchenko, E., Aliper, A. M., Makarev, E., Paz, K., Buzdin, A. A., et al. (2015). Pathway activation strength is a novel independent prognostic biomarker for cetuximab sensitivity in colorectal cancer patients. Hum. Genome Var. 2:15009. doi: 10.1038/hgv.2015.9

Conflict of Interest: $\mathrm{MS}, \mathrm{AGa}$, and $\mathrm{AB}$ have a financial relationship with OmicsWay Corp.

The remaining authors declare that the research was conducted in the absence of any commercial or financial relationships that could be construed as a potential conflict of interest.
The handling editor declared a shared affiliation with the authors AB, MS, and $\mathrm{AGu}$ at the time of review.

Copyright (C) 2021 Sorokin, Borisov, Kuzmin, Gudkov, Zolotovskaia, Garazha and Buzdin. This is an open-access article distributed under the terms of the Creative Commons Attribution License (CC BY). The use, distribution or reproduction in other forums is permitted, provided the original author(s) and the copyright owner(s) are credited and that the original publication in this journal is cited, in accordance with accepted academic practice. No use, distribution or reproduction is permitted which does not comply with these terms. 